\title{
Normatividad y visión societal en políticas públicas participativas de salud en Centroamérica
}

\author{
Ximena de los Ángeles Barros Rubio \\ Pontificia Universidad Católica de Chile
}

\author{
Victoria Soledad Rivera Ugarte \\ Universidad Alberto Hurtado
}

\begin{abstract}
Normatividad y visión societal en políticas públicas participativas de salud en Centroamérica
Resumen: Las políticas públicas en salud de Centroamérica incorporan la participación social como elemento fundamental para la optimización de los servicios de salud y la consecución de la equidad social. No obstante, en éstas, subyacen mecanismos que las debilitan, distorsionan, producen bajos niveles de participación, obstaculizan su promoción y profundizan el nivel central. El artículo analiza este tipo de políticas públicas en Guatemala, El Salvador, Nicaragua y Costa Rica, implicando categorías de análisis diferentes a los modos tradicionales de análisis de política pública: visión societal luhmanniana y normatividad habermasiana. Metodológicamente, se usa la investigación de tipo exploratoria documental de fuentes bibliográficas electrónicas. Como resultado, las políticas presentan elementos postconvencionales, que no operan en la implementación ni evaluación de la política, y la tendencia es hacia la jerarquía, por lo cual, el desafío es superar la presencia del "actor omnisciente".

Palabras clave: Participación social en salud. Normatividad. Visión de sociedad. Políticas públicas de salud.
\end{abstract}

Normatividade e visão social sobre as políticas públicas participativas da saúde na América Central Resumo: As Políticas de saúde pública na América Central incorporam a participação social como um elemento fundamental para otimizar os serviços de saúde e alcançar a equidade social. No entanto, existem mecanismos que enfraquecem, distorcem, produzem baixos níveis de participação, dificultam a sua promoção e aprofundam o nível central delas. O artigo analisa esse tipo de política pública na Guatemala, El Salvador, Nicarágua e Costa Rica, envolvendo diferentes categorias dos modos tradicionais de análise das políticas públicas: a visão societária de Luhmann e a normatividade de Habermas. Metodologicamente, se usa a pesquisa de tipo exploratório documental das fontes bibliográficas eletrônicas. Como resultado, as políticas apresentam elementos pôs convencionais que não atuam na implementação nem na avaliação da política e sua tendência no sentido da hierarquia, portanto, o desafio é superar a presença do "ator onisciente".

Palavras-chave: Participação social na saúde. Normatividade. Visão da sociedade. Políticas públicas de saúde.

\section{Normativity and Social Vision about Participatory Public Healthcare Policies in Central America}

Abstract: Public healthcare policies in Central America incorporate social participation as a fundamental elemental for improving healthcare services and achieving social equality. Nevertheless, there are mechanisms that weaken, distort, and produce low levels of participation and impede their promotion. The article analyzes this type of public policy in Guatemala, El Salvador, Nicaragua and Costa Rica, involving different categories of traditional modes of public policy analysis: Luhmann's societal view and Habermas' normativity. Methodologically, it uses exploratory documental research of electronic bibliographic sources. As a result, the policies present post-conventional elements that do not influence the implementation or the evaluation of policy and its tendency towards hierarchy, therefore, the challenge is to overcome the presence of the "conscious actor".

Keywords: Social participation in healthcare. Normativity. View of society. Public healthcare policies. 


\section{Introducción}

Dentro de los sistemas de salud, la participación social ${ }^{1}$ se ha constituido, desde inicios de los'90, como condición sinequanon de las políticas públicas en salud, siendo el pilar fundamental de la Reforma de Salud ${ }^{2}$ para Centroamérica y su búsqueda por reducir las desigualdades y favorecer la recuperación de la salud, utilizando de manera eficiente los recursos asociados (CONSEJO DE MINISTROS DE SALUD DEL SISTEMA DE LA INTEGRACIÓN CENTROAMERICANA, 2009, p. 7). Paradójicamente, diversas revisiones del tema (COMISIÓN NACIONAL DE SEGUIMIENTO REFORMA INTEGRAL DE SALUD, 2006, p. 12; KLIKSBERG, 2011; VÁSQUEZ et al., 2002) describen, tanto una brecha entre las políticas participativas en salud y su ejecución, como también una brecha entre los principios orientadores de las políticas de salud y los resultados de la participación social en los países de Centroamérica.

En consideración de esto, en este artículo se pretende observar este fenómeno mediante el modelo de análisis de políticas públicas desarrollado por Miranda Rebeco (2011, 2012), el que propone comprender la construcción de las políticas públicas a través de dos aspectos que dan forma (STEIN; TOMASSI, 2006) a las policies: una visión de la sociedad (LUHMANN, 1998) que remite a distintos estadios de diferenciación social y un tipo de normatividad, que explora los estadios de evolución de la moralidad, en principio desarrollados por Habermas (2008).

Por lo tanto, el objetivo del artículo es ofrecer un camino interpretativo mediante la visión de la sociedad luhmaniana y la normatividad habermasiana, desde donde se confrontará la semántica de las mismas ante los diferentes niveles de diferenciación social y de estadios de moralidad de las políticas participativas de salud, respectivamente. Para esta observación, se ha considerado trabajar con las políticas participativas en salud de cuatro países de Centroamérica: Guatemala, El Salvador, Nicaragua y Costa Rica.

\section{Normatividad y visión de sociedad en política pública como clave para la comprensión de la participación social}

Centroamérica se encuentra caracterizada por cifras alarmantes ${ }^{3}$ : el 41,41\% de su población es rural y el 50,9\% viven en situación de pobreza. Nicaragua presenta un 61,9\% de población en esa condición; Guatemala un 54,8\%; El Salvador un 47,9\%, mientras que la cifra más baja la tiene Costa Rica con un 18,9\%. Esto se refleja en el $\mathrm{PIB}^{4}$ donde Costa Rica septuplica a Nicaragua. Lo anterior, condiciona el Índice de Desarrollo Humano (IDH), donde Guatemala y Nicaragua se ubican en la posición 131 y 129, respectivamente, en contraste con Costa Rica, ubicado en el nivel 69 (ajustando el IDH, incluyendo la distribución de los alcances en educación, salud e ingresos). Referido a la esfera política ${ }^{5}$, Costa Rica ha tenido uno de los sistemas políticos más estables en comparación con Nicaragua, Guatemala y El Salvador, los que han experimentado regímenes militares, dictatoriales, intervencionismo por parte de E.E.U.U. y revueltas campesinas. Sin embargo, desde hace 20 años, estos países han fortalecido la democracia representativa de tipo presidencialista y elecciones libres, instalando políticas macroeconómicas neoliberales, abierta a mercados internacionales que ha aumentado el crecimiento en estas naciones, pero no ha redundado en mayor equidad e inclusión, en el mediano plazo.

Ante este panorama, la participación social se configura como la herramienta que permitiría superar las condiciones sociales adversas de la región, al imponerse a lo que Ocampo llama "pobres simbólicos", quienes más allá de la pobreza material, sufren de la "falta total de acceso al poder y las decisiones públicas" (OCAMPO, 2000, p. 302).

Por lo anterior, es imprescindible establecer que los mecanismos de participación incorporan al otro desde diferentes grados de incidencia para propender a su desarrollo e inclusión en el crecimiento de los países, lo que, para Corporación Participa, puede ir desde el acceso a la información como condición básica, la consultiva - en donde se distingue aquella que plantea la obligatoriedad de recibir una respuesta -, el nivel decisorio de incidencia en algunos ámbitos específicos, hasta, en su grado máximo, la co-gestión, la que según esta institución, implicaría un proceso de incidencia permanente en todas las fases de la política pública, desde el diseño hasta la evaluación (CORPORACIÓN PARTICIPA, 2008, p. 31-32).

Asimismo, las formas de articulación entre actores diversos, tanto privados, sociedad civil y la propia diversificación y especialización del aparato estatal, plantean nuevos desafíos para su coordinación (MASCAREÑO, 2010), en tanto la participación, como posibilidad de incidencia pública, se distribuye de manera heterogénea entre los diferentes sectores de la sociedad, como también entre los niveles nacionales y subnacionales, en donde la descentralización del poder surge como problemática esencial en esta materia (BOISIER, 2004). A la luz de esto y a nivel normativo, se puede señalar que la participación se entendería como un espacio permanente y transversal a la política pública, orientado a la deliberación y en donde los ciudadanos son reconocidos en tanto interlocutores válidos, con capacidad de tomar decisiones sobre asuntos 
que les afectan, a través de un diálogo simétrico, sin coacción y ponderando las consecuencias, en donde los actores centrales debieran ser los afectados por dichas decisiones (HABERMAS, 2000; RIVERA, 2010).

Los distintos niveles de participación posibles de observar en las políticas públicas de salud en Centroamérica serán abordados analíticamente mediante la indagación en la normatividad y visión de sociedad que subyacen a éstas, en tanto elementos que dan forma a las policies (STEIN; TOMMASI, 2006), permitiendo sostener, en mayor o menor medida, los procesos de participación social implicados en la relación Estadosociedad civil que están a la base del fortalecimiento de la democracia (CUNILL, 1995; OSZLAK, 1997).

En esa misma línea de fortalecimiento de la democracia mediante una resignificación en la relación Estado-sociedad, la participación social en salud es del todo consistente con lo que se ha desarrollado en párrafos anteriores. La declaración de Alma Ata indicó que la participación social era la estrategia que solucionaría los problemas de salud de la población, mediante los servicios de salud adecuados a las necesidades de la misma, actuando comprometida y responsable, identificando brechas y mejoras para dar continuidad a los cuidados de salud y satisfacer sus necesidad de manera deliberada y democrática (OPS, 1994).

Coherentemente, la normatividad de las políticas públicas dice relación con la validez de los procesos a partir de los principios que operan en las tomas de decisiones asociadas a las políticas públicas. Tomando los aportes de Habermas (1998), la validez estaría dada en la medida en que se incluyan los principios de diálogo utilización del habla como medio para llegar al entendimiento - y universalidad - capacidad de ponderar consecuencias, más allá del contexto inmediato - entre los actores afectados por la política. Para alcanzar este nivel de normatividad, Habermas sostiene que el actor debiera transitar por un proceso evolutivo donde, en primer lugar, se encuentra el nivel preconvencional de desarrollo moral, caracterizado por una perspectiva egocéntrica en la que, en el primer estadio, opera una moralidad heterónoma, donde los actores responden a poderes externos mediante el castigo y la obediencia. En su segundo estadio, el egocentrismo tiene ahora una orientación moral dada por fines instrumentales, de intercambio e interés individual. Para la política pública, implicaría formas de participación cuya motivación sería la obtención de beneficios personales o bien, evadir castigos de cualquier tipo (penales, tributarios, simbólicos etc.). El segundo nivel de desarrollo moral es el convencional, apareciendo las expectativas interpersonales mutuas, la motivación y la acción moral por acuerdos interpersonales según un grupo de referencia. Ejemplos de políticas públicas de este tipo serían aquellas en las que la motivación a la participación sería el logro de un objetivo de un grupo de interés específico, sin la consideración de las consecuencias para grupos a los que el actor no pertenece. Finalmente, en el nivel post convencional, el más alto en términos de desarrollo moral, predomina una perspectiva universalista y formal, basada en un contrato social entre los actores que componen una sociedad, ya sea respetando acuerdos universales propio del quinto estadio, como abriendo el diálogo para la construcción de nuevos acuerdos en el sexto y más alto estadio. Sería, en el nivel post convencional, el nivel que favorecería el despliegue de altos estándares de participación social (RIVERA, 2010), manifestados en la habilitación social, participación de diversos actores en la toma de decisiones, acumulación de capital social y empoderamiento (SERRANO, 1998).

Sin embargo, la normatividad - representada por el argumento pragmático universal (HABERMAS, 1987) en donde el horizonte ideal y contrafáctico es desplegado en comunidades concretas - estará circunscrita a una determinada visión de sociedad, la que genera el marco en donde los principios, en este caso, vinculados a la participación dialógica y universal, se desplegarán. Aquí se pueden considerar los aportes de Luhmann (2007), quien señala que la sociedad ha pasado por diferentes estadios de diferenciación social - segmentaria, estratificada y funcional -, proceso que presiona a que las políticas públicas abandonen el carácter jerárquico y asuman, en su operar, el policentrismo dominante propio de sociedades modernas (LUHMANN, 2007).

Esto implica que la visión de sociedad de las políticas públicas debiera estar abierta a la inclusión de la diversidad, tanto de actores sociales como de escenarios y elementos intervinientes. Como sostiene Aguilar, en el contexto actual "el gobernar difícilmente podrá ser exitoso si el gobierno sigue usando sus embotados instrumentos de intervención dirigista y alineamiento político, por lo que hay que descubrir o construir el nuevo formato e instrumental directivo que permita a una sociedad tener sentido y capacidad de dirección, alcanzar metas superiores y ampliar su horizontes" (AGUILAR, 2006, p. 40).

A partir de estas dos referencias teóricas - normatividad y visión de sociedad - es posible generar un cruce "desde donde emergen, al modo de los weberianos, tipos ideales" (MIRANDA REBECO, 2011, p. 141), encontrando los siguientes: a) diferenciación segmentaria/normatividad preconvencional, donde converge "la igualdad de los sistemas parciales de la sociedad" (LUHMANN, 2007, p. 485) - donde cada individuo tendrá una posición determinada en el orden social - con una motivación de la acción basada en perspectivas particularistas de comportamiento (HABERMAS, 2008); b) diferenciación estratificada/normatividad convencional, tipología en la que se avanza en una diferenciación social jerárquica, cuyo centro es el sistema político, a la que corresponde una normatividad convencional, primando principios que responden a un orden social particular y una ética que se ajusta a la ley (HABERMAS, 2008); c) diferenciación funcional/normatividad 
posconvencional, caracterizado por una visión de sociedad policéntrica o heterárquica (LUHMANN, 2007), sin un centro prioritario y cuya normatividad corresponde a la postconvencional, en donde priman una ética de la intención y de la responsabilidad junto a un derecho formal.

En términos generales, este marco asume que en las sociedades modernas las políticas debieran responder a una visión de sociedad heterárquica, donde lejos de constituirse un centro estratégico con jerarquía para observar y transformar la sociedad, sería un polo más, operando entre otros sistemas de igual nivel. Esto entregaría las condiciones para que opere un alto desarrollo de la normatividad de dichas políticas, haciendo factible que la legitimidad esté dada por la participación dialógica de los actores afectados por la política.

No obstante, cabe reconocer que este mismo proceso y su normatividad subyacente se confronta a un contexto tensionado por instituciones estratégicamente orientadas según medios y fines (HABERMAS, 1987), y en donde, incluso, la universalidad en el acceso a los derechos presenta contradicciones frente a un contexto neoliberal que ha determinado la acción de las políticas (OSZLAK, 1997; HINKELAMMERT, 2005). Al respecto, Offe (1994, p. 136) señala que este sistema, si bien surge como respuesta a las consecuencias del liberalismo, presentándose como un "modelo casi universalmente aceptado para crear cierta medida de paz y armonía social en las sociedades europeas de postguerra se ha convertido desde los setenta en fuente de nuevas contradicciones y divisiones políticas". En la misma línea, Habermas (1988, p. 72) sostiene que este contexto ha puesto en jaque la legitimidad misma del Estado, en la medida en que "el programa del Estado social, que sigue alimentándose de la utopía de la sociedad del trabajo, ha perdido la capacidad de formular posibilidades futuras de alcanzar una vida colectiva mejor y más segura". De ahí la importancia de problematizar la dimensión normativa de las políticas, la visión de sociedad implicadas y los espacios de participación que es capaz de sostener.

\section{Metodología}

A partir de las perspectivas teóricas señaladas, el artículo busca describir analíticamente las tendencias de normatividad y visión de sociedad que subyacen a las políticas de participación social en salud en Centroamérica y que condicionan sus resultados. Se seleccionaron cuatro países, Guatemala, El Salvador, Nicaragua y Costa Rica, bajo dos criterios: disponibilidad de fuentes secundarias; y características disímiles entre sí en materia de trayectorias históricas sobre temas de participación. El análisis se hizo en base a una investigación de tipo exploratoria documental, ya que las fuentes consultadas corresponden a textos, fuentes bibliográficas, electrónicas, de reconocida experticia en el tema de salud pública y para ello, se usaron dos matrices de análisis, basadas en indicadores que levantaron elementos componentes de la normatividad y visión de sociedad subyacente a las políticas participativas en salud.

\section{Participación social en políticas públicas de salud en Guatemala, El Salvador, Nicaragua y Costa Rica: tendencias desde la normatividad y visión de sociedad}

\subsection{Normatividad de las políticas participativas de salud en Centroamérica: asentada en estadios convencionales con elementos post convencionales en la enunciación}

En términos generales, las políticas públicas de salud en estos países emergen desde los niveles centrales de la orgánica gubernamental, en este caso, en los respectivos Ministerios de Salud. A saber, el caso de Costa Rica, el Ministerio de Salud se encarga de la: "dirección y conducción; regulación del desarrollo de la salud; vigilancia de la salud e investigación y desarrollo tecnológico" (SÁENZ et al., 2011, p. 53). Igual situación ocurre en Nicaragua, donde el Ministerio de Salud es rector del sector salud y responsable de elaborar, aprobar, aplicar, supervisar y evaluar normas técnicas y formular las políticas, planes, programas, proyectos, manuales e instructivo (MARTÍNEZ, 2010, p. 15). En ninguno de estos países se reconoce la centralidad de lo local o de las comunidades en el diseño de políticas públicas.

En Guatemala y El Salvador, si bien la Constitución atribuye al Ministerio de Salud Pública y Asistencia Social una función rectora, las reformas descentralizadoras han generado una segmentación del sistema nacional de salud (BECERRIL-MONTEIKO, 2011; LÓPEZ DÁVILA, 2011, p. 205), pero no conducente a un involucramiento ciudadano (OPS, 2007, p. 25).

Los elementos participativos tienden a ser explícitos en todos los países indagados. En Nicaragua, la participación ciudadana es un derecho constitucional (MUISER; SÁENZ; BERMÚDEZ, 2011, p. 237), reforzado por el contexto de la reforma del sistema de salud y de la Ley de Participación (LEY DE PARTICIPACIÓN 
CIUDADANA, 2003), en donde los diversos agentes sociales inciden e interactúan bajo el Modelo Atención de Salud Familiar y Comunitario ${ }^{6}$, iniciado el 2007 y que opera a través de los Sistemas Locales de Atención Integral en Salud ${ }^{77}$ de forma descentralizada y gratuita. Así, se reorienta la participación ciudadana desde un enfoque utilitario hacia la gestión ciudadana de salud (OPS, 2002, p. 19).

El Salvador acaba de estrenar una Política Nacional de Participación Social en Salud en febrero del año 2013, donde explícitamente se indica "fortalecer la organización social y el involucramiento ciudadano en temas sanitarios" (COMISIÓN NACIONAL DE SEGUIMIENTO A LA PROPUESTA DE REFORMA INTEGRAL DE SALUD, 2006, p. 5) para responder a la necesidad de que el ejercicio democrático contribuya en la toma de las decisiones ante un sistema de salud salvadoreño centralista, excluyente y restrictor (ACOSTA et al., 2011).

En Costa Rica, la implementación del Modelo de Salud Familiar y Comunitario (en la atención primaria de salud) y la Ley de Descentralización de los Hospitales y Clínicas desean alcanzar niveles de participación social, pero no resultan del todo vinculante (SÁENZ et al., 2011).

En Guatemala, la participación social se manifiesta en los programas de promoción emanados desde el Departamento de Promoción y Educación en Salud ${ }^{8}$. Durante los años 2000-2005, se capacitaron los Consejos Municipales y Consejos Comunitarios de Desarrollo, definiendo la figura de guardianes de la salud, agentes comunitarios encargados de transmitir información desde y hacia las familias ${ }^{9}$. No obstante, esto no necesariamente se tradujo en estrategias que aumentaran la participación social, sino más bien, manifestaron una falta de voluntad política para volverla una realidad institucional.

Ahora bien, la motivación de estos países para contemplar la participación social en salud puede responder a imperativos externos o a lineamientos intrínsecos del país como un fin democrático. Para Nicaragua, la participación social en salud proviene del reconocimiento explícito y taxativo de éste manifestado en su propia agenda y en la Ley de Participación.

Para El Salvador, los elementos de participación social provienen de las recomendaciones internacionales y, por ello, forman parte de su sistema de salud: "la participación social ha sido parte del contenidos de las recomendaciones y compromisos internaciones que han suscritos los países de Las Américas [...] y que, a su vez forman parte de los componentes estratégicos de los diversos enfoques integrales en salud" (COMISIÓN NACIONAL DE SEGUIMIENTO A LA PROPUESTA DE REFORMA INTEGRAL DE SALUD, 2006, p. 7).

Para Costa Rica, la participación social en salud es estratégica dentro del proceso de producción nacional de servicios de salud, por lo que se presupone la intervención de actores sociales en dicho proceso. Para Costa Rica, "la salud de la población no se produce solamente desde los servicios de salud asistenciales, sino que es el resultado del accionar conjunto de los actores sociales en interacción con su medio ambiente (...), para esto se requiere de una acción concreta y eficaz de la sociedad en el establecimiento de prioridades, la adopción de decisiones, la planificación de estrategias y su implantación, con el fin de mejorar la salud" (MINISTERIO DE SALUD DE COSTA RICA, 2008, p. 23).

Por último, para Guatemala, más allá de su fuerte componente educativo y según la Ley de Consejos de Desarrollo Urbano y Rural, la participación social a nivel interinstitucional es un bien fundamental que considera la articulación con las instituciones formales de producción de salud a nivel gubernamental (GUATEMALA, 2002).

Como se ha revisado, todos los países reconocen la necesidad de la participación social en salud y la incorporan efectivamente como política pública. Sin embargo, ¿Qué tipología de participación social se está promoviendo? En general, se observa que la relación vinculante con la ciudadanía aún no es abordada mediante mecanismos ad hoc para ello. Por ejemplo, en Costa Rica, si bien gozan de un planificación sanitaria que buscar tener una ciudadanía informada, organizada e involucrada en el diseño e implementación de programas sanitarios, los canales institucionales permiten únicamente formular sugerencias y reclamos a los mismos (SÁENZ et al., 2011, p. 164).

En Guatemala, si bien el Código de Salud garantiza la participación de la población en la definición, implementación y evaluación de las políticas públicas de salud, en la práctica no existen mecanismos explícitos y viables para que la ciudadanía ejerza esa participación. Es por eso que las formas de participación social en salud (voluntariado y organizaciones sociales prestadoras de servicios de salud) se ponen al servicio de políticas públicas ya definidas (BECERRIL-MONTEIKO, 2011; LÓPEZ DÁVILA, 2011, p. 206).

En El Salvador, si bien tienen una política de participación social en salud "todavía existe un desarrollo parcial (...) y se dificulta por la fragmentación del sistema de salud (...) en temas importantes como: promoción de la salud, participación de los ciudadanos en salud, evaluación y promoción del acceso equitativo a los servicios de salud" (OPS, 2007, p. 31).

Por último, en Nicaragua, el nivel de injerencia de la participación social en la gestión de salud ha buscado fortalecerse en lo local, a través del Consejo Nacional de Salud: “(...) se han conformado los Consejos de Salud con un grado diverso de participación ciudadana (de acuerdo a las características de cada Región Autónoma del Atlántico) y además se cuenta con la participación de las delegaciones de las distintas instituciones 
gubernamentales de acuerdo a lo que establece la Ley General de Salud" (OPS, 2009, p. 38). Sin embargo, eso no necesariamente se traduce en una participación social vinculante ${ }^{10}$, así como también se observa un predominio de actores estatales por sobre los ciudadanos.

En virtud de lo anterior, desde el año 2000, la OPS monitorea a los todos los países de la región centroamericana, en relación al comportamiento de las autoridades sanitarias y la participación social en salud, utilizando las llamadas Funciones Esenciales de Salud Pública (FESP) ${ }^{11}$. Sin perjuicio de lo anterior, en Nicaragua, la Política Nacional de Salud 2008 incorpora elementos evaluativos de la política de participación social en salud, enfatizando la importancia de la participación de los municipios y comunidades en los procesos de evaluación a través de los SILAIS. En Costa Rica, en tanto, "cuenta con instrumentos, leyes, normas, políticas y planes para el desempeño óptimo de las FESP. El desafío es fortalecer las estrategias que permitan su implementación y fiscalización, así como la definición de metas claras que identifiquen qué es importante vigilar, cada cuánto y con qué recursos" (OPS, 2009, p. 24), pero no hace explícito el lugar que ocupan las comunidades, ciudadanos y organizaciones locales en este proceso.

En el caso de El Salvador, si bien la FESP hace una buena evaluación respecto de elementos centrales para las políticas de salud como monitoreo, evaluación, investigación y control de riesgos y daños de salud pública; desarrollo de políticas y capacidad institucional de planificación y gestión en salud pública, fortalecimiento de la capacidad institucional de regulación y fiscalización en salud pública (OPS, 2007, p. 37), no hay mayor información de resultados en relación a la participación social en salud. Lo mismo ocurre en Guatemala, donde el Ministerio de Salud, entidad gubernamental encargada de evaluar las acciones de salud, se orienta, más bien, a evaluar planes y programas de directa incidencia sanitaria, como productos farmacéuticos, inocuidad de alimentos, acreditación de establecimientos de salud (OPS, 2007, p. 27), pero no se evidencia presencia de una preocupación especial por la evaluación de las políticas de participación en salud.

Las encuestas de satisfacción usuaria son, también, mecanismos evaluativos que reportan información en torno a la gestión de calidad de los servicios de salud, desde el punto de vista de los usuarios. Si bien no cumplen con una función de cogobierno, al menos, demuestran la valoración que tienen los usuarios del sistema de salud, constituyéndose en un eje informativo para el mismo. Este mecanismo es ampliamente usado en Nicaragua y Costa Rica (SÁENZ et al., 2011, p. 165), no así en Guatemala y El Salvador, donde esta experiencia parece no ser sistemática por parte del Ministerio de Salud, respectivamente ${ }^{12}$.

\subsection{Visión de sociedad de las políticas participativas de salud: una constelación jerárquica en Centroamérica}

En Centroamérica, las políticas son diseñadas, implementadas y evaluadas desde una lógica jerárquica: en la cima, los tomadores de decisiones del nivel central (ministerios y organismos internacionales); en el medio, los implementadores, como departamentos e instancias de alcance territorial; abajo, los usuarios de las políticas. Antecedentes de esto se pueden encontrar Nicaragua, donde pese al proceso de descentralización iniciado el año 2004 e impulsado desde el gobierno central de Nicaragua y la Comisión Nacional Interinstitucional, la participación se reduce a instituciones gubernamentales, ya que no integra mayormente a actores externos. En Costa Rica, queda explícita la combinación jerarquía/heterarquía en la siguiente definición del sistema nacional de salud: "sector salud (está) conformado por el conjunto de instituciones públicas centralizadas y descentralizadas, que hacen una competencia explícita y legal dirigida a proveer servicios de salud a la población" (OPS, 2009, p. 25). De esa manera, pese a tener instituciones descentralizadas (CCSS, A y A y INS) ${ }^{13}$ con potestad para manejar su financiamiento y su gasto en autonomía, es el Ministerio de Salud quien debe mandatar y orientar las líneas de inversión y gasto en salud.

El Salvador busca avanzar hacia un desarrollo heterárquico, pero "los esfuerzos tendientes a modernizar e integrar las instituciones sectoriales, a descentralizar la gestión (...) han encontrado vacíos legales, organizativos y resistencias políticas y no han logrado el consenso necesario que permitan que el SS transite hacia la integración sistémica" (OPS, 2007, p. 29).

Para Guatemala, los grandes procesos encaminados hacia la heterarquía se han manifestado en proyectos descentralizadores de la toma de decisiones, como es el caso de los Consejos de Desarrollo Urbano y Rural que reciben un 10\% del presupuesto estatal para la ejecución de proyectos de desarrollo local (GUATEMALA, 2002).

Asimismo, los esfuerzos señalados se alinean con el interés por integrar la participación y enfoque comunitario, expresándose en el Modelo de Salud Familiar Comunitario que se implementa en Costa Rica, a través de los EBAIS, y en Nicaragua, con los SILAIS, el modelo Incluyente en Salud que se implementa en Guatemala mediante los SIAS ${ }^{14}$ y los ECOS ${ }^{15}$ Familiares y Especializados en El Salvador. En el caso de Nicaragua, el Modelo de Salud Familiar contempla la acción de los SILAIS y Hospitales, los cuales cuentan con las Juntas Directivas, donde participan actores sociales, instituciones y entidades autónomas. Territorialmente, esto constituye un esfuerzo 
relevante por acercar los servicios de salud a las comunidades, relevante para un país donde las distancias son determinantes en el acceso a la salud. No obstante, en términos políticos, el poder que adquieren estos estamentos se limita a la administración. Además, existe coordinación con organismos locales compuestos por voluntarios, que se encargan de asuntos donde los EBAIS ${ }^{16}$ no tienen alcance (SERRANO, 2001, p. 28).

La situación en Costa Rica, a través de los $\mathrm{EBAIS}^{17}$, también constituyó un avance en materia de heterarquía, pero que, a su vez, presenta importantes elementos jerárquicos que aminora la polaridad. Los EBAIS se instalaron como parte del proceso de reforma a la salud que experimentaba el país para cubrir servicios de atención primaria. Al poco andar, las comunidades más vulnerables del país aumentaron la demanda por estos equipos y, mediante la organización social, lograron favorecer la cobertura en toda la dispersión territorial de Costa Rica, mejorando los resultados macrosanitarios del país. Con todo lo anterior, igualmente persisten problemas de financiamientos originado por la cobertura financiera de quienes no están asegurados, pero tienen el derecho universal y garantizado de estarlo.

\section{O Respecto a la visión de}

$$
\text { sociedad, hay una tendencia }
$$

hacia la jerarquía, dada su centralización en los

Ministerios de Salud de cada

uno de los países revisados, y

en donde los ciudadanos

tienen posibilidades

restringidas de operar frente

al fuerte centralismo de la

orgánica estatal y su

burocracia, tanto en la

formulación de políticas como

en su control.

Con respecto a Guatemala, existe una visión crítica de lo que las SIAS están contribuyendo, pues la amplia participación de las comunidades y sus organizaciones está ausente en todo el proceso y se evidencia una importante carencia en el enfoque intercultural de la prestación de servicios, en un país cuya mayoría es indígena (VERDUGO, 2000, p. 60). Corresponde señalar que las SIAS son una manifestación de descentralización del modelo de gestión en salud y de la focalización de éste en población con altos índices de vulnerabilidad; no obstante, sigue siendo formulado a nivel central por lo que no es posible articularlo a las características locales. Al momento de planificar las acciones que se llevarán a cabo, no se contemplan los aspectos locales como dispersión comunitaria, diferentes condiciones de salud para adecuar la oferta sanitaria a lo que realmente se presenta como problemática de salud: más bien se busca acomodar la rigidez de la planificación centralista a las particularidades locales, lo que constituye un factor de jerarquía.

Para El Salvador, los procesos de reforma en salud y, por consiguiente, de descentralización, ha dejado sin coordinación los tres subsectores de salud: público (representado por los Sistemas Básicos Integrados de Salud), privado (representado por ONG's, empresa privada y de cohorte intercultural e indígena) y sanidad militar (CEPAL, 2000, p. 155). Ante este panorama, una solución jerárquica, como fortalecer el nivel central, fue sugerida por parte del Ministerio de Salud. Asimismo, como se mencionó anteriormente, se encuentran insertos en el sistema de salud sólo aquellos que se encuentran incluidos en el sistema laboral formal. Para quienes son campesinos independientes y miembros de cooperativas, se superponen las desventajas de estar excluidos, tanto del sistema laboral formal como de salud y de la concentración territorial de los recursos en zonas urbanas ${ }^{18}$.

Con respecto a la diversidad indígena o intercultural como parte constitutiva de una sociedad, ésta es reconocida, pero no operacionalizada, es decir, no se traduce en acciones concretas. Salvo en el caso de El Salvador y Guatemala, donde sí reconocen formalmente, en las políticas públicas de salud, a la medicina con raigambre indígena, como una alternativa válida al sistema formal.

\section{Conclusiones y reflexiones finales}

Desde una exploración, a partir de los paradigmas de normatividad y visión de sociedad (MIRANDA REBECO, 2011, 2012), que subyacen a las políticas de salud en los cuatro países analizados, es posible señalar que en éstas priman visiones jerárquicas de sociedad y una normatividad convencional que limitarían la inclusión de actores diversos y, por lo tanto, de su participación social.

Desde la normatividad, puede observarse que en las políticas de salud de la región centroamericana existen elementos postconvencionales en la enunciación, pero que no operan en la implementación y evaluación 
de la política. Con esto, el espacio de articulación de las políticas públicas se encuentra limitado por quienes son los tomadores de decisiones pertenecientes a las burocracias estatales. Lo anterior limita las posibilidades de procesos dialógicos con la ciudadanía, a fin de co-construir un sistema de salud que haga sentido a las necesidades experimentadas y que esté acorde a las particularidades de actores y contextos diversos. Éstos serían más bien operadores de una política de diseño centralizado.

Respecto a la visión de sociedad, hay una tendencia hacia la jerarquía, dada su centralización en los Ministerios de Salud de cada uno de los países revisados, y en donde los ciudadanos tienen posibilidades restringidas de operar frente al fuerte centralismo de la orgánica estatal y su burocracia, tanto en la formulación de políticas como en su control. Desde allí, se determinan los roles y funciones de cada actor en el sistema de salud, pero sin reconocer de manera suficiente la autonomía de los diferentes actores y escenarios con los que converge.

Es rescatable, no obstante, que el actual desarrollo normativo como la visión de sociedad preponderante, es fruto de las reformas de salud impulsadas en la región en las décadas de los ' 90 y 2000 y su énfasis por promover la descentralización tanto administrativa como de la toma de decisiones, por lo que se espera que dichos esfuerzos en el futuro tiendan a reconocer la heterogeneidad de las sociedades modernas, permitiendo que las políticas de salud superen la idea de constituirse como un 'actor omnisciente' o 'tutor moral de la sociedad'.

\section{Referencias}

ACOSTA, M. et al. Sistema de salud de El Salvador. Salud Pública Méx., v. 53, supl. 2, p. 188-196, 2011.

AGUILAR, L. Gobernanza y Gestión Pública. México: Fondo de Cultura Económica, 2006.

BECERRIL-MONTEIKO, V.; LÓPEZ DÁVILA, L. Sistema de Salud de Guatemala. Salud Pública Méx., v. 53, supl. 2, p. 197-208, 2011.

BOISIER, S. Desarrollo territorial y descentralización. El desarrollo en el lugar y en las manos de la gente. Revista Eure, Santiago de Chile, v. XXX, n. 90, p. 27-40, sept. 2004. Disponible en: <http://www.scielo.cl/pdf/eure/v30n90/art03.pdf>. Acceso en: 19 agosto 2014.

BOSSERT, T.; BOWSER, D.; COREA, L. Studies of Decentralization of the Health System in Nicaragua: Final Report. Harvard School of Public Health, 2001.

CEPAL. Las reformas de salud en América Latina y el Caribe: su impacto en los principios de la seguridad social. Capítulo III: El impacto de las reformas de salud en los principios de seguridad social. Documentos de proyectos, 2000.

Indicadores sociales baìsicos de la subregioin norte de Ameìrica Latina y el Caribe. Edicioìn 2008-2009. 2009. Disponible en: <http://www.cepal.org/publicaciones/xml/7/38107/L947.pdf>. Acceso en: 20 agosto 2014.

COMISIÓN NACIONAL DE SEGUIMIENTO A LA PROPUESTA DE REFORMA INTEGRAL DE SALUD. Propuesta Política de Participación Social en Salud. San Salvador, El Salvador, mayo, 2006.

CONSEJO DE MINISTROS DE SALUD DEL SISTEMA DE LA INTEGRACIÓN CENTROAMERICANA. Agenda de salud de Centroamérica y República Dominicana, 2009-2018. Unidos por la salud de nuestros pueblos. 2009.

CORPORACIÓN PARTICIPA. Manual de participación ciudadana. Santiago, Junio, 2008.

CUNILL, N. La rearticulación de las relaciones Estado-Sociedad: en búsqueda de nuevos sentidos. Revista del CLAD Reforma y Democracia, Caracas, n. 4, p. ¿, 1995.

DE LEÓN, O.; ARTEAGA, O.; SCHIATFINO, I. Medición del nivel de satisfacción de usuarios que asisten al Centro de Salud de Acatenango, Guatemala. Rev Chil Salud Pública, v. 7, n. 2, p. 80-87, 2003.

MINISTERIO DE SALUD DE COSTA RICA. Modelo Conceptual y Estratégico de la Rectoría de la Producción Social de la Salud. Marco Estratégico Institucional. San José, Costa Rica. 14 de marzo 2008. Disponible en: <http://www.ministeriodesalud.go.cr/ sobre_ministerio/do/productos/VI\%20Cont.\%20En\%2009\%20a\%20Ab\%2010/Productos\%20Componente\%20de\%20capacitaci\%3Fn, Febrero\%202009-abril\%202010/Modulo\%202B/contenidos/s3/m2-s3-d1.pdf>. Acceso en: 20 agosto 2014.

HABERMAS, J. Teoría de la acción comunicativa. Madrid: Taurus, 1987.

La crisis del Estado de bienestar y el agotamiento de las energías utópicas. Ensayos políticos. Madrid: Ed. Península, 1988. Facticidad y validez. Madrid: Trotta, 1998.

Aclaraciones a la ética del discurso. Madrid: Trotta, 2000.

Conciencia moral y acción comunicativa. Madrid: Trotta, 2008.

HINKELAMMERT, F. La transformación del Estado de Derecho bajo el impacto de la estrategia de globalización. Polis - Revista Latinoamericana, n. 10, 2005. Disponible en: <http://polis.revues.org/7482>. Acceso: 20 agosto 2014.

KLIKSBERG, B. Estrategias y metodologías para promover la participación social en la definición e implantación de políticas públicas de combate a las inequidades en salud. 2011. Disponible en: <http://cmdss2011.org/site/wp-content/uploads/2011/07/ Participaci\%C3\%B3n-Social_Bernardo-Kliksberg.pdf>. Acceso en: 20 agosto 2014.

GUATEMALA. Ley de Consejos de Desarrollo Urbano y Rural. Decreto n. 11. 2002. Disponible en: <http://sistemas.segeplan.gob.gt/ discode/sche\$portal/documentos/ley_concejos_desarrollo_guatemala.pdf > . Acceso en: 20 agosto 2014. 
LEY DE PARTICIPACIÓN CIUDADANA. Ley n. 475. Aprobada el 22 de Octubre del 2003. Disponible en: <http:// legislacion.asamblea.gob.ni/Normaweb.nsf/(\$All)/F78CA467F5C96D0306257257005FBADC?OpenDocument>. Acceso en: 20 agosto 2014.

LUHMANN, N. Complejidad y modernidad. Madrid: Trotta, 1998.

. La sociedad de la sociedad. México: Herder, 2007.

MARTÍNEZ, J. Sistemas de atención médica en Centroamérica: estudio comparativo sobre su capacidad para enfrentar la crisis actual. Informe final. 2010. Disponible en: 〈http://www.estadonacion.or.cr/files/biblioteca_virtual/centroamerica/004/Matinez_2010.pdf〉. Acceso en: 20 agosto 2014.

MASCAREÑO, A. Coordinación social mediante políticas públicas: el caso chileno. Revista CEPAL, n. 101, 2010.

MIRANDA REBECO, P. (en coautoría con Aldo Mascareño, Alejandro Pelfini y Gabriela Rubilar): Instituciones formales y redes informales en políticas públicas: Interferencias procesuales y normativas en políticas de pobreza, obras públicas y medioambiente en Chile. 2012. Disponible en: http://gigapp.org/administrator/components/com_jresearch/files/publications/WP-2012-10.pdf >. Acceso en: 20 agosto 2014.

Tensiones estructurales y semánticas en las políticas públicas. Una cartografía del caso chileno. Textos \& Contextos, Porto Alegre, v. 10, n. 1, p. 137-156, jan./jul. 2011.

MUISER, J.; SÁENZ, M. R.; BERMÚDEZ, J. L. Sistema de salud de Nicaragua. Salud Pública Méx., v. 53, supl. 2, p. 233-242, 2011. MUÑOZ, F. et al. Las funciones esenciales de la salud pública: un tema emergente en las reformas del sector de la salud. Rev Panam Salud Pública, Washington DC, v. 8, n. 1-2, Jul./Ago. 2000.

OFFE, C. Contradicciones en el Estado del bienestar. Madrid: Alianza, 1994.

OCAMPO, J. Equidad, desarrollo y ciudadanía. Cepal, agosto 2000.

OPS - Organización Panamericana de la Salud. Evaluacioin para el fortalecimiento de procesos de participacioin social en la promocioin y el desarrollo de la salud en los sistemas locales de salud. Washington D.C.: OPS, 1994.

_. Análisis de las Reformas del Sector Salud en la Subregiónde Centroamérica y la República Dominicana. Washington D.C.: OPS, 2002

Perfil de sistemas de salud de Guatemala. Washington, D.C.: OPS, 2007.

2007.

. Perfil del sistema de salud de El Salvador 2000-2005: monitoreo y análisis de los procesos de cambio. Washington, D.C: OPS,

. Perûl de los Sistemas de Salud Costa Rica. Washington, D.C.: OPS, 2009.

. Perfil de Sistemas de Salud en Nicaragua: Monitoreo y Análisis de los Procesos de Cambio y Reforma. Washington, D.C.: OPS,

2009.

OSZLAK, O. Estado y sociedad: ¿nuevas reglas de juego? Revista del CLAD Reforma y Democracia, Caracas, n. 9, 1997.

. Segundo informe sobre desarrollo humano en Centroamérica y Panamá. Proyecto Estado de la Regioìn. San Joseì, Costa Rica, 2003. Disponible en: 〈http://www10.iadb.org/intal/intalcdi/PE/2011/09374.pdf>. Acceso en: 20 agosto 2014.

RIVERA, V. Participación como espacio de deliberación: desafíos para la política pública. Revista de Trabajo Social, Pontificia Universidad Católica de Chile, n. 79, p. 49-61, 2010.

SÁENZ, M. R. et al. Sistema de salud de Costa Rica. Salud Pública Méx., v. 53, supl. 2, p. 156-167, 2011.

SERRANO, C. Participación social y ciudadanía.Un debate del Chile contemporáneo. 1998. Disponible en: <www.asesoriasparaeldesarrollo.cl>. Acceso en: 20 agosto 2014.

SERRANO, A. (Comp.). La democracia y sus desafíos en Nicaragua. Fundación Friedrich Ebert, Universidad Politécnica de Nicaragua, Centro Interuniversitario de Estudios Latinoamericanos y Caribeños "Mauricio López”, 2001.

STEIN, E.; TOMMASI, M. La política de las políticas públicas. Política y gobierno, México, v. XIII, n. 2, II semestre, 2006.

VÁSQUEZ, M. L. et al. Los procesos de reforma y la participación social en salud en América Latina. Gaceta Sanitaria, España, v. 16, n. 1, p. 30-38, 2002.

VERDUGO, J. C. Análisis sobre la reforma del sector salud en Guatemala. Análisis de la implementación del modelo de primer nivel de atención del sistema integral de atención en salud (SIAS) en Guatemala. Documento técnico de Estudio de 3 implementadoras del SIAS de Primer Nivel de Atención en Salud, Estudio financiado por Memisa, Holanda. Guatemala, abril 2000.

\section{Notas}

1 A partir de los lineamientos de Alma Ata, la participación en salud se focaliza en la atención primaria, tomando en cuenta la ventaja comparativa que tiene al estar inserta en una comunidad definida territorialmente y espacialmente, siendo una estrategia efectiva considerando que es la comunidad la que mejor puede responder y adecuar la oferta sanitaria a sus efectivas necesidades.

2 Los procesos de Reforma a los Sistemas de Salud se comenzaron a implementar en Centroamérica a partir de la década de ' 80 y ' 90 . Estos estaban orientados a buscar la flexibilidad y adecuación de la oferta a las necesidades de la población, mediante la descentralización y la participación de los ciudadanos. Para mayor profundización ver Vásquez et al. (2002).

3 Datos obtenidos de Cepal (2009). 
4 Producto Interno Bruto.

5 Datos obtenidos de PNUD (2003).

6 Siglas: MOSAFC.

7 Siglas: SILAIS. Los Sistemas Locales deAtención Integral en Salud otorgan servicios de salud gratuitos con atención focalizada en población en situación de pobreza, enfatizando la prevención, promoción, recuperación y rehabilitación en salud en municipios urbanos y rurales. Para mayor información ver Bossert, Bowser e Corea (2001).

8 Dependiente administrativamente del Ministerio de Salud de Guatemala.

9 Los agentes comunitarios llamados guardianes de la salud adquieren completo sentido, considerando la baja adhesión y cobertura que tiene la población, en su conjunto, con respecto al sistema nacional de salud, cercano al $40 \%$.

10 Cabe señalar que la participación social en Nicaragua ha sido elogiada por el PNUD en el año 2009, precisamente por la experiencia de voluntariado que se desarrolla en políticas sociales de salud y educacional y por la mayor inclusión política de los pueblos indígenas, Para profundizar en esta reseña ver: www.un.org/spanish/News.

11 Para mejorar la práctica de salud pública, se requiere contar con los instrumentos para valorar su estado actual y las áreas en las que debe ser fortalecida. Es por eso que la Organización Panamericana de la Salud(OPS) ha puesto en marcha la iniciativa "La salud pública en las Américas", dirigida a la definición y medición de las FESP como base para mejorar la práctica de la salud pública y fortalecer el liderazgo de las autoridades sanitarias en todos los niveles del Estado. Para mayor información consultar Muñoz et al.

12 Sin embargo, cabe rescatar que en Guatemala se ejecutó un estudio de evaluación de satisfacción usuaria conocido como: De León, Arteaga e Schiatfino (2003). No obstante no subsana la falta de aplicación de este instrumento en la población usuaria.

13 Caja Costarricense del Seguro Social, Acueducto y Alcantarillado e Instituto Nacional de Seguros, respectivamente.

14 Sistema Integral de Atención en Salud.

15 ECOS Familiar: Equipos Comunitarios de Salud Familiar; ECOS Comunitarios: Equipos Comunitarios de Salud Familiar Especializados.

16 Equipo Básico de Salud.

17 Los Equipos Básicos de Atención Integral en Salud están compuestos por un médico general, un auxiliar de enfermería y un asistente técnico de atención primaria en salud, brindando servicios en clínicas o centros de salud en el territorio.

18 Y la mayor parte de la proporción rural del país conforma parte importante del $40 \%$ de personas que viven bajo la línea de la pobreza.

\title{
Ximena de los Ángeles Barros Rubio
}

xdbarros@uc.cl

Magíster en Ciencia Política de la Pontificia Universidad Católica de Chile

Asesora Técnica en Servicio de Salud Metropolitano Suroriente - Ministerio de Salud de Chile

\section{Pontificia Universidad Católica de Chile}

Avenida Vicuña Mackenna, 4860, Macul

Región Metropolitana, Chile

\section{Victoria Soledad Rivera Ugarte}

victoriaru@gmail.com

Magíster en Trabajo Social de la Pontificia Universidad Católica de Chile

Docente del Departamento de Trabajo Social de la Universidad Alberto Hurtado

\author{
Universidad Alberto Hurtado
}

Calle Cienfuegos, 41, Santiago Centro

Región Metropolitana, Chile 